\title{
Echolucent carotid plaque is associated with restenosis after carotid endarterectomy
}

\author{
*Qing Li, MD,' Beibei Liu, MD,' Yue Zhao, MD,, Yumei Liu, MD, PhD, ${ }^{1}$ Mingjie Gao, MD, PhD, \\ Lingyun Jia, MD, PhD, ${ }^{1}$ Liqun Jiao, MD, PhD, ${ }^{2}$ and Yang Hua, MD ${ }^{1,3}$

\begin{abstract}
Departments of ${ }^{1}$ Vascular Ultrasound and ${ }^{2}$ Neurosurgery, Xuanwu Hospital, Capital Medical University, Beijing, China; and ${ }^{3}$ Center of Vascular Ultrasonography, Beijing Institute of Brain Disorders, Beijing, China
\end{abstract}

\begin{abstract}
OBJECTIVE The mechanism of carotid endarterectomy (CEA) restenosis remains unclear. Our research aimed to investigate the relationship between the carotid plaque grayscale median (GSM) value and restenosis after CEA.

METHODS Between January 2010 and January 2018, 1280 consecutive patients underwent CEA at our institution; 32 patients were diagnosed with restenosis by ultrasound at 1 year after CEA. The correlations between plaque GSM, plaque echogenicity, clinical manifestations, shunting, and restenosis were analyzed.

RESULTS In total, 829 patients were ultimately enrolled; 32 (4\%) presented diagnoses of restenosis (mean age 67.3 \pm 8.0 years, $81.2 \%$ men). The GSM value was lower in the restenosis group $(68.1 \pm 19.9$ vs $59.9 \pm 14.7, p=0.02)$. After multiple logistic regression analysis, the GSM value was found to be an independent risk factor for restenosis (OR $0.976,95 \% \mathrm{Cl} 0.957-0.995)$. Shunting was another significant independent risk factor for restenosis (OR 2.39, 95\% $\mathrm{Cl} 1.07-5.34$ ). The GSM cutoff value for predicting restenosis was 75 (sensitivity 0.38 , specificity 0.84 , area under the curve 0.62). We separated the patients into 2 groups by GSM (GSM $\leq 75$ and GSM $>75$ subgroups). Comparison of the 2 groups indicated that symptomatic manifestation was related to restenosis in the subgroup with GSM $\leq 75$, indicating predominantly echolucent plaques, but not in the subgroup with GSM > 75, indicating predominantly echogenic plaques.
\end{abstract} CONCLUSIONS Predominantly echolucent carotid plaques, as measured by GSM, had a higher restenosis risk at 1 year than echogenic plaques.

https://thejns.org/doi/abs/10.3171/2020.2.JNS193397

KEYWORDS carotid endarterectomy; carotid plaque; grayscale median; restenosis; ultrasound; vascular disorders

$\mathrm{C}$ AROTID artery stenosis is one of the major causes of stroke. Carotid endarterectomy (CEA) has been widely accepted as the gold standard operation for carotid artery stenosis since the 1990 s. ${ }^{1}$ However, restenosis is one of the long-term complications after CEA and is correlated with stroke recurrence. The rate of restenosis differs among various randomized controlled trials, ranging from $3 \%$ to $10 \%{ }^{2-6}$ based on distinct diagnostic standards and follow-up periods.

Some researchers have reported that female sex, diabetes mellitus, and dyslipidemia may be risk factors for restenosis, ${ }^{7}$ whereas others have indicated that age was the only risk factor. ${ }^{2}$ Pathologists have elucidated a possible relationship of carotid plaque, which is filled with smooth muscle cells, and CEA restenosis. ${ }^{8}$ As mentioned in a previous literature review, clinical risk factors and pathological components of plaque may be causes of restenosis after CEA, though the final conclusions and mechanisms remain controversial. Additionally, pathological component analysis of the plaque must be conducted on affected individuals. It would be beneficial to distinguish a specific in vivo indication that can predict restenosis after CEA. Carotid atherosclerosis can manifest as intraplaque hemorrhage and calcification. Katano and Yamada ${ }^{9}$ quantified carotid plaque calcification to analyze the relationship between the calcium score and restenosis 1 year after CEA surgery. ${ }^{10}$ Their study reminds us that other methods can be used to quantify carotid plaque to investigate its correlation with restenosis.

Vascular ultrasound, a noninvasive and convenient de-

ABBREVIATIONS AUC = area under the curve; CEA = carotid endarterectomy; DSA = digital subtraction angiography; $\mathrm{GSM}=$ grayscale median; ICA = internal carotid artery; nIMT = neointima-media thickness; PSV = peak systolic velocity; ROC = receiver operating characteristic.

SUBMITTED December 17, 2019. ACCEPTED February 4, 2020.

INCLUDE WHEN CITING Published online April 17, 2020; DOI: 10.3171/2020.2.JNS193397.

${ }^{*}$ Q.L. and B.L. contributed equally to this work. 
vice, plays an irreplaceable role in carotid screening and follow-up studies. The present study was designed to investigate the relationship of ultrasound carotid plaque grayscale median (GSM) quantification with CEA restenosis after a 1-year follow-up.

\section{Methods \\ Patients}

This retrospective cohort study was approved by the ethics committee of our hospital. The study began in January 2010 and ended in January 2018, and during this period 1280 consecutive patients underwent CEA at Xuanwu Hospital. Neurosurgeons performed the standard CEA surgery on patients who had carotid stenosis of $\geq 70 \%$ and patients who were symptomatic (<6 months) with 50\%$69 \%$ stenosis that had been confirmed by CTA or digital subtraction angiography (DSA). The vascular ultrasound team performed the preoperative routine GSM estimation on all participants. Assessments were conducted immediately (assessment within 1 week after CEA surgery); at 3, 6 , and 12 months; and annually after 1 year. Monitoring was also performed as part of the routine examinations by the vascular ultrasound team.

\section{Inclusion Criteria}

The inclusion and exclusion criteria were as follows. Included patients where those with 1$)$ severe stenosis $(\geq 70 \%)$ or who were symptomatic ( $<6$ months) with $50 \%-69 \%$ stenosis verified by CTA or DSA before surgery; 2) completion of preprocedural color Doppler flow imaging estimation; and 3) completion of immediate assessment and follow-up examinations at 3, 6, and 12 months. Excluded patients had 1) absence of baseline information or ultrasound estimation; 2) unsuccessful endarterectomy recanalization due to acute thrombosis; 3 ) $\geq 50 \%$ residual stenosis; 4) loss to follow-up; or 5) heavy calcification and acoustic shadow plaque for which the GSM could not be measured.

\section{Ultrasound Machine and Sonographer}

Ultrasound systems with $4.0-8.0 \mathrm{MHz}$ linear transducers and 2.0-5.0 MHz convex array probes were used (Philips IU22, Koninklijke Philips NV, and Hitachi Ascendus). All of the examinations were performed by experienced sonographers (with $>5$ years of experience). Training courses on how to collect the standard images and save them to the picture archiving and communication system (PACS) were organized for the sonographers.

\section{Ultrasound Criteria of Stenosis and Restenosis}

All of the participants were assessed by use of the stenosis criteria of the North American Symptomatic Carotid Endarterectomy Test (NASCET) method. ${ }^{1}$ Stenosis (70\%-99\%) was defined as a stenosis site peak systolic velocity (PSV) of $>230 \mathrm{~cm} / \mathrm{sec} .{ }^{11}$ Since there is no consensus on the definition of restenosis, our institution adopted the primal stenosis criterion as the restenosis diagnosis criterion according to the NASCET method. Restenosis (50\%-69\%) was defined as a stenosis site PSV of 125-230 $\mathrm{cm} / \mathrm{sec}$. An additional essential criterion was that the ratio of the PSV at the stenosis site ( $\left.\mathrm{PSV}_{\text {stenosis site }}\right)$ to the PSV 4-6 $\mathrm{cm}$ beyond the bifurcation level of the carotid artery $\left(\mathrm{PSV}_{\text {distal }}\right)$ was 2.0-4.0.

Restenosis (70\%-99\%) was defined as a $\mathrm{PSV}_{\text {stenosis site }}$ of $>230 \mathrm{~cm} / \mathrm{sec} \cdot{ }^{11}$ An additional essential criterion was that the ratio of the $\mathrm{PSV}_{\text {stenosis site }}$ to the $\mathrm{PSV}_{\text {distal }}$ was $>4.0$.

\section{Carotid Plaque Imaging}

The narrowest section of the carotid artery caused by carotid plaque in the longitudinal and transverse sections was imaged. The echo characteristics detected by $2 \mathrm{D}$ ultrasound were classified into the following 5 groups based mainly on the percentage of echogenic carotid plaque ${ }^{12,13}$ occupying the carotid artery: type I: uniformly echolucent, $<15 \%$ echogenic; type II, predominantly echolucent, 15\%-50\% echogenic; type III: predominantly echogenic, $50 \%-85 \%$; type IV: uniformly echogenic, $>85 \%$; and type V: plaques that could not be classified into the other 4 groups because of heavy calcification and acoustic shadow.

We categorized type I and II plaques into the predominantly echolucent group and type III and IV plaques into the predominantly echogenic group. We excluded patients with type $\mathrm{V}$ plaques. We recorded the stenosis sites caused by the carotid plaque, which included the common carotid artery, carotid bulb, and internal carotid artery (ICA).

\section{GSM Measurement}

Image J (Java-based image processing software developed at the National Institutes of Health) was used to adjust the narrowest longitudinal section to the standardized image. The areas of the standardized image that referred to the area representing blood had a gray value of zero, while the brightest area of the adventitia had a gray value of 190. We created an algebraic linear curve that adjusted the primal GSM variable for each plaque to a standardized GSM value that is the true value of each plaque. We circled the plaque outline, recorded the GSM value, and calculated the standardized GSM value of each plaque by using the algebraic linear curve. . $^{13,14}$ The 2 technicians who had received the standard training on GSM measurement were blinded to the trial and results. They sketched the plaque outline and calculated the GSM values separately, back to back. The final GSM was the average of the values determined by the 2 technicians (Fig. 1).

\section{Endarterectomy Procedure}

Every patient first signed a consent form. Preoperative aspirin $(100 \mathrm{mg} /$ day $)$ was routinely administered to every participant at least 5 days before the operation; for the patients who had aspirin intolerance, the aspirin was substituted with clopidogrel $(75 \mathrm{mg} /$ day). CEA was performed by experienced neurosurgeons who had sufficient vascular surgery training. All patients received general anesthesia, and the CEA procedure was adopted in a standard fashion according to the published literature. ${ }^{15}$ Throughout the operation, all patients were monitored by transcranial Doppler monitoring (TCD) and routine electrocardiograph monitoring. Shunting was determined to be necessary if TCD monitoring indicated that the velocity of the middle cerebral artery decreased $>50 \%$ after clamping 


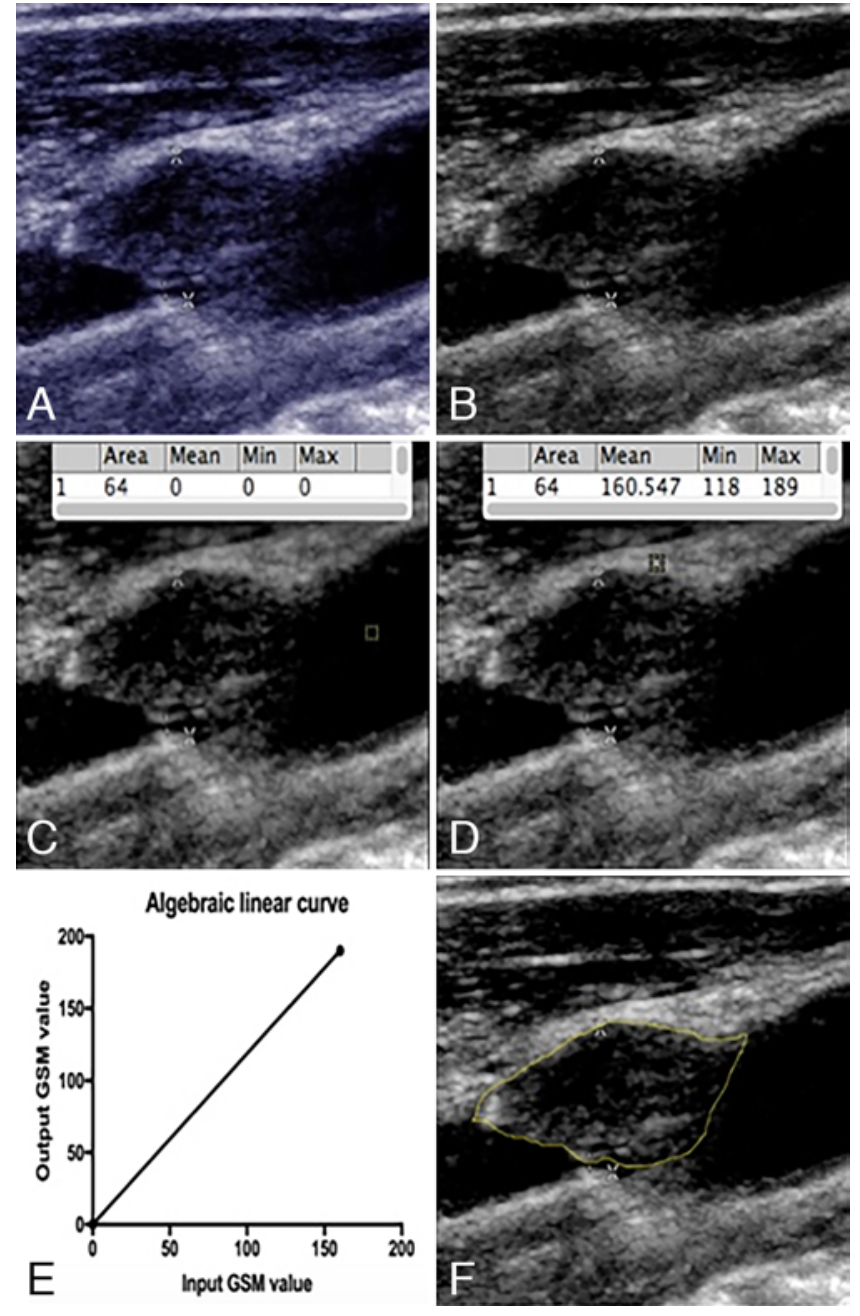

FIG. 1. GSM measurement procedure. A: Culprit plaque in B-ultrasound. B: Culprit plaque in ImageJ, standardized version. C: Selecting blood area for a grayscale of zero while avoiding noise. D: Selecting adventitia membrane for a grayscale of 190. E: Generating the algebraic linear curve. F: Sketch of the plaque outline, measurement of the GSM, and calculation of the adjusted GSM value of the plaque by using an algebraic linear curve. Figure is available in color online only.

of the ICA. The need for patch and tacking sutures was determined at the neurosurgeons' discretion. Antiplatelet medications and statins were administered for long-term maintenance after CEA surgery.

\section{Statistical Analysis}

Normally distributed continuous variables are expressed as means \pm standard deviations. Skewed distributions of continuous variables are expressed as medians (interquartile ranges), and categorical variables are expressed as numbers (percentages). For equivalent variables with a normal distribution, the independent Student t-test was utilized to compare the 2 patient groups. The Mann-Whitney U-test was used to compare categorical variables and abnormally distributed variables between the 2 groups. Receiver operating characteristic (ROC) curve analysis was performed to find the optimal cutoff value to predict restenosis. Areas under the curve (AUCs), optimal threshold values, sensitivity, and specificity were calculated. A logistic regression analysis was performed to screen the independent risk factors. A 2-sided $p$ value $<0.05$ was considered statistically significant; confidence intervals were set at $95 \%$. Statistical analyses were performed by using IBM SPSS (version 22.0) and GraphPad Prism (version 6.01) software.

\section{Results}

\section{Patient Demographic Characteristics and Plaque Characteristics in the 2 Groups}

In total, 829 patients were ultimately enrolled in the study; 32 cases of 1-year restenosis were estimated by ultrasound (Fig. 2, study flowchart), of which 20 cases had $50 \%-69 \%$ restenosis and 12 cases had $70 \%-99 \%$ restenosis. The age distribution was $65.9 \pm 7.9$ years. Clinical features and plaque characteristics, as well as detailed descriptions of the operations, are summarized in Table 1. Patients with restenosis had lower GSM values $(59.9 \pm$ 14.7 vs $68.1 \pm 19.9, \mathrm{p}=0.02$ ), a higher frequency of echolucent plaques $(93.8 \%$ vs $55.7 \%, \mathrm{p}<0.001)$, a higher frequency of shunting $(28.1 \%$ vs $13.7 \%, p=0.022)$, a higher frequency of symptomatic clinical manifestations $(56.2 \%$ vs $28.2 \%, \mathrm{p}=0.001$ ), and a lower frequency of smoking history $(53.1 \%$ vs $69.5 \%, p=0.05)$.

\section{GSM Cutoff Value for Restenosis}

A comparison of the significance and risk of restenosis at different GSM values using the relative risk model was employed to analyze the role of different GSM cutoff points (Fig. 3A, Supplemental Table 1). The ROC curve was used to determine the cutoff value of GSM to identify patients at higher risk of restenosis at 1 year; a GSM value of 75 was the threshold (sensitivity 0.38 , specificity 0.84 , AUC 0.62, Youden's index 0.22; Fig. 3B).

\section{Comparison of Restenosis in Different GSM Subgroups}

Table 2 presents detailed descriptions of restenosis by subgroups defined according to the meaningful factors screened according to Table 1 and GSM levels. Symptomatic manifestations and shunting were predominantly responsible for increasing the risk of restenosis in the GSM $\leq 75$ group rather than in the GSM $>75$ group.

\section{Multiple Logistic Regression Analysis of GSM and Restenosis}

As shown in Table 3, the GSM value was a meaningful factor for predicting restenosis in the unadjusted analysis. The association remained after adjusting for all confounders (OR 0.976, 95\% CI 0.957-0.995). After we classified the GSM value into 2 groups by using 75 as the threshold, GSM $\leq 75$ was generally seen as a stronger factor related to restenosis compared to GSM > 75 in the unadjusted analysis (OR 2.86, 95\% CI 1.10-7.49). The relationship still made a strong contribution to predicting restenosis in the fully adjusted model (adjusted OR 2.86, 95\% CI 1.10-7.52). Echolucent plaque had a notable impact on the risk of restenosis before and after the adjusted analysis (Table 3 ). The 


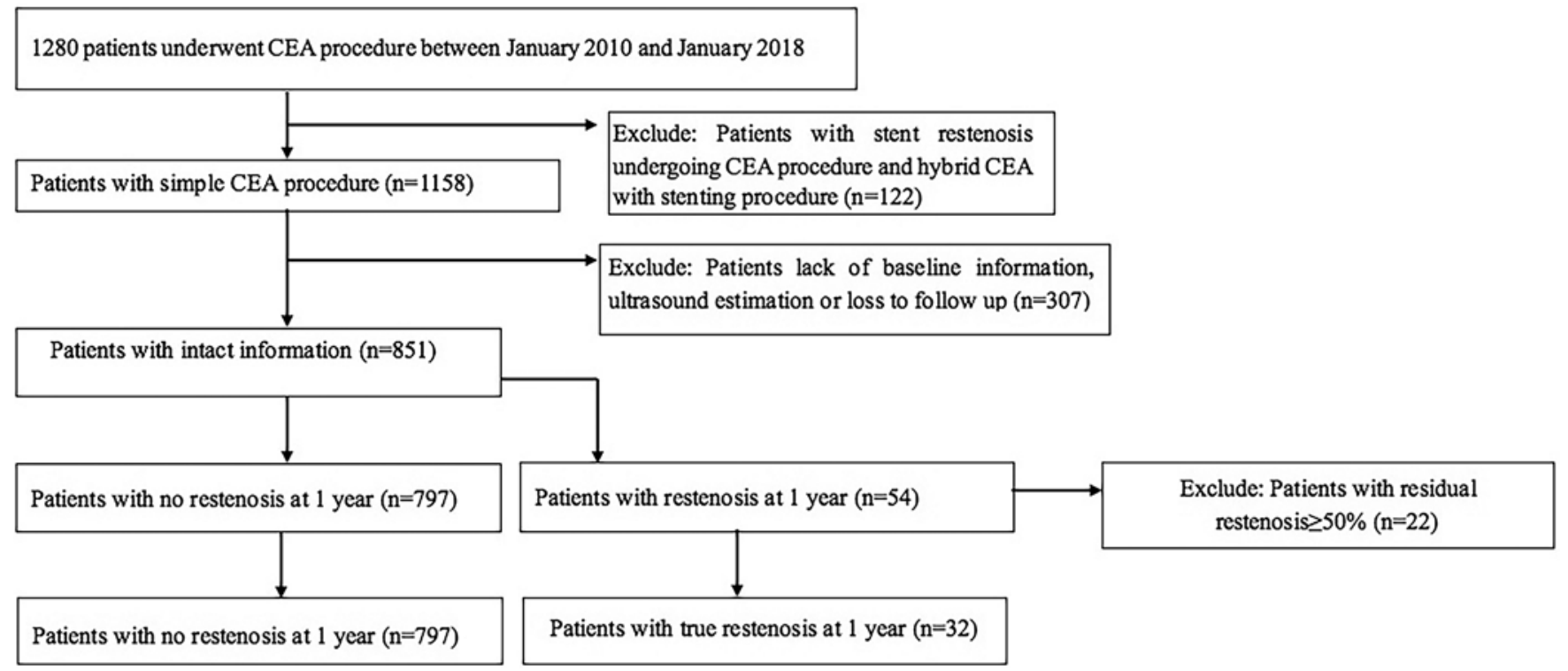

FIG. 2. Study flowchart.

TABLE 1. Patient demographic features and risk factors

\begin{tabular}{|c|c|c|c|}
\hline & $\begin{array}{l}\text { Controls } \\
(n=797)\end{array}$ & $\begin{array}{l}\text { Restenosis }>50 \% \\
\qquad(n=32)\end{array}$ & $\mathrm{p}$ Value \\
\hline Male & $686(86.1)$ & $26(81.2)$ & 0.442 \\
\hline Age in yrs & $68.1 \pm 8.0$ & $67.3 \pm 8.0$ & 0.769 \\
\hline Hypertension & $555(69.6)$ & $22(68.8)$ & 0.915 \\
\hline Diabetes mellitus & $238(29.9)$ & $13(40.6)$ & 0.194 \\
\hline Coronary heart disease & $151(18.9)$ & $3(9.4)$ & 0.172 \\
\hline Dyslipidemia & $393(49.3)$ & $14(43.8)$ & 0.537 \\
\hline Smoking history & $554(69.5)$ & $17(53.1)$ & 0.05 \\
\hline Symptomatic & $225(28.2)$ & $18(56.2)$ & 0.001 \\
\hline Op side, right & $437(54.8)$ & $17(53.1)$ & 0.849 \\
\hline Op type, eversion & $29(3.6)$ & $1(3.1)$ & 0.879 \\
\hline Shunting & $109(13.7)$ & $9(28.1)$ & 0.022 \\
\hline Patch & $19(2.4)$ & $1(3.1)$ & 0.789 \\
\hline Echolucent plaque & $444(55.7)$ & $30(93.8)$ & $<0.001$ \\
\hline GSM value & $68.1 \pm 19.9$ & $59.9 \pm 14.7$ & 0.02 \\
\hline Plaque length (mm) & $18.7 \pm 7.3$ & $22.8 \pm 8.1$ & 0.464 \\
\hline Plaque thickness (mm) & $3.3 \pm 1.3$ & $3.9 \pm 1.1$ & 0.360 \\
\hline Plaque calcification & $491(61.6)$ & $21(65.6)$ & 0.646 \\
\hline Ulceration & $172(21.6)$ & $3(9.4)$ & 0.097 \\
\hline Stenosis site, ICA & $732(91.8)$ & $26(81.2)$ & 0.075 \\
\hline Postop evaluation & & & 0.573 \\
\hline Normal & $774(97.1)$ & 30 (93.8) & \\
\hline $\begin{array}{c}<50 \% \text { residue } \\
\text { stenosis }\end{array}$ & $23(2.9)$ & $2(6.2)$ & \\
\hline
\end{tabular}

Values are presented as number (\%) of patients or mean \pm SD unless otherwise indicated. effects of symptomatic clinical manifestations and smoking history disappeared after adjustment for all confounders. Shunting was another significant independent risk factor for restenosis (adjusted OR 2.39, 95\% CI 1.07-5.34).

\section{Neointima-Media Thickness at 1 Year in the Different GSM Subgroups}

We analyzed the relationship between GSM and neointima-media thickness (nIMT). Figure 4 presents the linear correlation between these variables $(\mathrm{r}=-0.125, \mathrm{p}<$ 0.001).

\section{Discussion}

Our research was intended to evaluate the relationship between the echogenicity of carotid plaque, as measured by the GSM, and restenosis at 1 year after CEA. The results indicated that GSM was an independent risk factor for restenosis. Although the effect was relatively weak, after dividing the 2 patient groups by using the GSM threshold of 75, lower GSM was found to increase restenosis risks, which suggests that patients with predominantly echolucent carotid plaques might have a higher restenosis risk after the CEA procedure than patients with echogenic plaques.

The underlying mechanism of echolucent carotid plaque-induced restenosis is still unknown; however, some reasons for this occurrence could be obtained from other studies. Intraplaque hemorrhage and a larger lipid core are known to manifest as echolucent, while calcification manifests as echogenic in B-ultrasound imaging. ${ }^{6,17}$ Hellings et al. ${ }^{18}$ analyzed the restenosis plaques extracted from patients who underwent a second CEA surgery and demonstrated that macrophage cell infiltration and a lipid core were associated with late restenosis. Katano et al. ${ }^{10}$ measured the CT value of the carotid plaque in vivo and reported that calcified plaque had a lower restenosis rate 

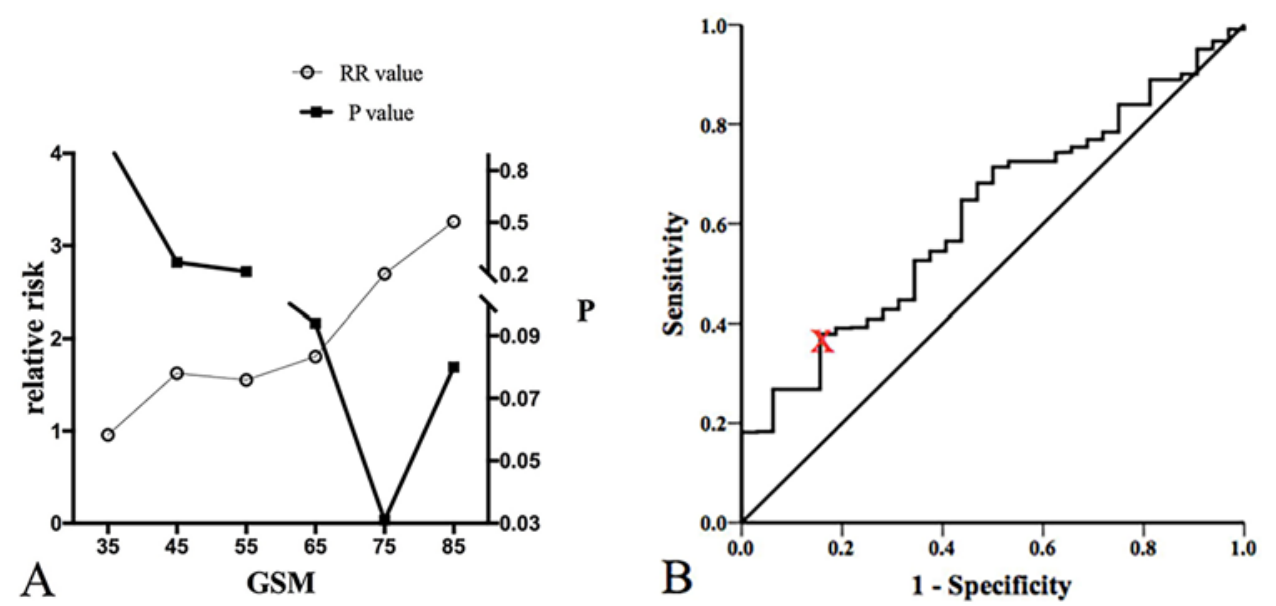

FIG. 3. Analysis of different GSM cutoffs to identify patients with a higher risk of restenosis at 1 year. A: Comparison of significance and relative risk of restenosis at 1 year using a relative risk model to analyze the role of different GSM cutoff values. B: $\mathrm{ROC}$ curve showing the optimal threshold of this method. Figure is available in color online only.

than noncalcified plaque. These investigators considered that the calcified component might inhibit neointima hyperplasia and protect the physical barrier from surgical injury. In our study, we measured the nIMT at 1 year after CEA, and there was a mild negative linear correlation between the GSM value and nIMT, which may partially explain the different restenosis results in the GSM subgroups. Takami et al..$^{19}$ focused on the relationship between carotid plaque components and the rate of nIMT increase after CEA. nIMT had an irreversible increase in patients with echolucent carotid plaque, whereas it had a temporary increase and slow decline in patients with echogenic plaque. Our result was consistent with this discovery by Takami et al., ${ }^{19}$ but the nIMT rate increase during the first year has to be confirmed by further observation.
Bonati et al. ${ }^{3}$ reanalyzed the CAVATAS (Carotid and Vertebral Artery Transluminal Angioplasty Study) data and reported that a history of smoking and current smoking were predictors of restenosis after endovascular therapy or CEA surgery. In our research, we analyzed the relationship between smoking history and restenosis and concluded that smoking history might be correlated with restenosis protection. One possible explanation is that smoking history in our research included patients who had already quit smoking, which might have obscured the results. Arquizan et al. ${ }^{2}$ reported that age was the only reason for restenosis. Other relevant factors, such as female $\operatorname{sex}^{20}$ and dyslipidemia, were also considered, but in our research, no difference associated with these confounders was detected.

In our research, we also found that symptomatic mani-

TABLE 2. Comparison of restenosis in the patient GSM subgroups

\begin{tabular}{|c|c|c|c|c|c|c|}
\hline & \multicolumn{2}{|c|}{ Overall } & \multicolumn{2}{|c|}{ GSM $\leq 75$} & \multicolumn{2}{|c|}{ GSM $>75$} \\
\hline & No. $(\%)$ & $p$ Value & No. $(\%)$ & $p$ Value & No. $(\%)$ & $p$ Value \\
\hline \multicolumn{7}{|l|}{ GSM } \\
\hline$\leq 75$ & $27 / 553(4.9)$ & 0.031 & - & - & - & - \\
\hline$>75$ & $5 / 276(1.8)$ & & - & & - & \\
\hline \multicolumn{7}{|c|}{ Plaque character } \\
\hline Echolucent & $30 / 474(6.3)$ & $<0.001$ & $26 / 432(6.0)$ & 0.019 & $4 / 42(9.5)$ & $<0.001$ \\
\hline Echogenic & $2 / 355(0.6)$ & & $1 / 121(0.8)$ & & $1 / 234(0.4)$ & \\
\hline \multicolumn{7}{|c|}{ Smoking history } \\
\hline Yes & $17 / 571(3.0)$ & 0.050 & $13 / 377(3.4)$ & 0.022 & 4/194 (2.1) & 0.619 \\
\hline No & $15 / 258(5.8)$ & & $14 / 176(8.0)$ & & $1 / 82(1.2)$ & \\
\hline \multicolumn{7}{|l|}{ Symptomatic } \\
\hline Yes & $18 / 243(7.4)$ & 0.001 & $15 / 158(9.5)$ & 0.001 & $3 / 85(3.5)$ & 0.153 \\
\hline No & $14 / 586(2.4)$ & & $12 / 395(3.0)$ & & $2 / 191(1.0)$ & \\
\hline \multicolumn{7}{|l|}{ Shunting } \\
\hline Yes & $9 / 118(7.6)$ & 0.022 & $8 / 71$ (11.3) & 0.007 & $1 / 47(1.8)$ & 0.861 \\
\hline No & $23 / 711(3.2)$ & & 19/482 (3.9) & & $4 / 229(1.7)$ & \\
\hline
\end{tabular}


TABLE 3. Multivariate regression analysis of the association between GSM and restenosis

\begin{tabular}{|c|c|c|c|c|}
\hline Exposure & Crude OR $(95 \% \mathrm{Cl})$ & OR $(95 \% \mathrm{Cl})$ Model $1^{*}$ & OR $(95 \% \mathrm{Cl})$ Model $2 \dagger$ & OR $(95 \% \mathrm{Cl})$ Model $3 \ddagger$ \\
\hline GSM & $0.976(0.958-0.995)$ & $0.976(0.957-0.995)$ & $0.975(0.956-0.995)$ & $0.976(0.957-0.995)$ \\
\hline \multicolumn{5}{|l|}{ GSM category } \\
\hline$\leq 75$ & $2.86(1.10-7.49)$ & $2.89(1.11-7.55)$ & $2.87(1.10-7.51)$ & $2.86(1.10-7.52)$ \\
\hline$>75$ & 1.00 (ref) & 1.00 (ref) & 1.00 (ref) & 1.00 (ref) \\
\hline \multicolumn{5}{|l|}{ Plaque character } \\
\hline Echolucent plaque & $11.82(2.82-49.53)$ & $12.23(2.91-51.39)$ & $12.30(2.92-51.84)$ & $13.21(3.11-56.11)$ \\
\hline Echogenic plaque & 1.00 (ref) & 1.00 (ref) & 1.00 (ref) & 1.00 (ref) \\
\hline \multicolumn{5}{|l|}{ Clinical manifestations } \\
\hline Asymptomatic & 1.00 (ref) & 1.00 (ref) & 1.00 (ref) & 1.00 (ref) \\
\hline Symptomatic & $3.14(1.56-6.32)$ & $0.98(0.95-1.03)$ & $0.42(0.19-0.94)$ & $1.06(0.14-7.95)$ \\
\hline Shunting & $2.45(1.13-5.29)$ & $2.51(1.15-5.51)$ & $2.38(1.07-5.26)$ & $2.39(1.07-5.34)$ \\
\hline Smoking history & $0.50(0.25-1.01)$ & $0.98(0.94-1.02)$ & $0.97(0.95-0.99)$ & $0.88(0.11-6.74)$ \\
\hline
\end{tabular}

${ }^{*}$ Adjusted for age and sex.

† Adjusted for age, sex, hypertension, diabetes mellitus, coronary heart disease, dyslipidemia, smoking, symptomatic manifestation, GSM value, and plaque characteristics.

$\ddagger$ Adjusted for age, sex, hypertension, diabetes mellitus, coronary heart disease, dyslipidemia, smoking, symptomatic manifestation, GSM value, plaque characteristics, operation type, patch, and shunting.

festation correlated with restenosis after carotid surgery in the lower GSM group, whereas it did not have an effect in the higher GSM group. The relationship between symptoms and restenosis might hypothetically be explained by the underlying plaque morphology since previous research demonstrated that symptomatic patients had a larger lipid core and greater macrophage infiltration, whereas there were fewer smooth muscle cells in culprit plaques. ${ }^{21}$ An abundant lipid core and high macrophage infiltration manifested as lower GSM values and the finding of echolucent formations under ultrasound examination. ${ }^{17}$ Since we had no pathological analysis of our culprit plaques, we could only infer the relationship between the culprit plaque pathological form and symptom manifestation, which remains to be verified by further research.

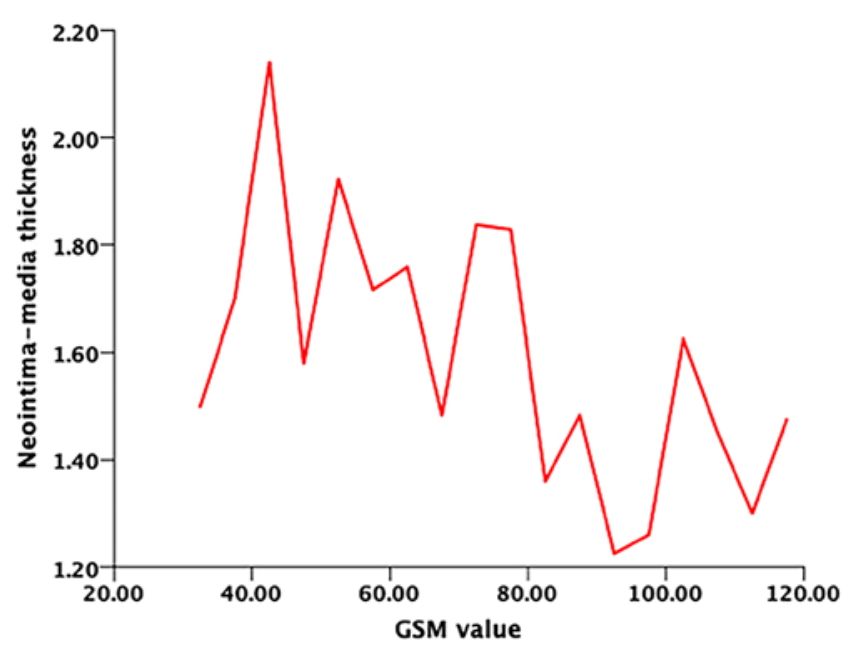

FIG. 4. Association between the GSM and nIMT values. Figure is available in color online only.
Previous researchers have presented the opinion that the use of shunting during surgery might induce damage to the intima-media barrier and stimulate neointima hyperplasia. ${ }^{22}$ In our research, we discovered that shunting was significantly correlated with restenosis in the fully adjusted model, especially in patients with echolucent plaque, which was consistent with the previous study.

We measured the carotid plaque in vivo by ultrasound to predict the restenosis risk in order to provide a method for physicians to identify higher-risk patients who may need close follow-up. We were only able to elucidate the relationship of a GSM $\leq 75$ with a higher restenosis risk than that for GSM > 75. However, we could not refuse to perform the CEA procedure in the higher-risk patients and instead opted for carotid artery stenting for them because we could not ensure that patients with a GSM $\leq 75$ would receive more benefit from a surgery other than CEA. This consideration needs to be investigated further in case-control observations and clinical trials.

The clinical function of measuring GSM by ultrasound was to identify a large number of patients (553 of 829, $66.7 \%$ ) at high risk of restenosis after CEA, especially to identify the subgroups of patients with higher restenosis rates compared to those for the overall patient population.

\section{Study Limitations}

Several limitations of this study should be clarified. First, our study was a single-center retrospective study that may have been subject to collection bias and recall bias. A larger prospective study should be conducted. Second, due to the limitations of the GSM measurement method, plaques with heavy calcification and acoustic shadows were excluded in our research, because they may have biased the results and weakened the function of calcified plaque. Third, the reproducibility of GSM measurement in our single center was high, but it was difficult to assure the absolute consistency 
of this method, especially in the context of multicenter research. However, our research provided evidence that carotid plaque morphology might be considered one factor that influences the risk of restenosis. Finally, we did not apply a pathological analysis in our research, which should be applied to supplement future studies.

\section{Conclusions}

Our research demonstrated that predominantly echolucent carotid plaques, as indicated by $\mathrm{GSM} \leq 75$, had a higher restenosis risk at 1 year than predominantly echogenic plaques, indicated by GSM > 75. Preoperational carotid plaque GSM measurements estimated by ultrasound could provide a functional predictive indicator for CEA surgery.

\section{Acknowledgments}

This study was supported by the Capital's Funds for Health Improvement and Research (2018-2-2011) awarded to Dr. Yang Hua. Qiujie Zhang and Yanli Song provided technical help in circling the plaque and measuring the GSM value in this research.

\section{References}

1. Barnett HJ, Taylor DW, Eliasziw M, et al. Benefit of carotid endarterectomy in patients with symptomatic moderate or severe stenosis. North American Symptomatic Carotid Endarterectomy Trial Collaborators. $N$ Engl J Med. 1998;339(20):1415-1425.

2. Arquizan C, Trinquart L, Touboul PJ, et al. Restenosis is more frequent after carotid stenting than after endarterectomy: the EVA-3S study. Stroke. 2011;42(4):1015-1020.

3. Bonati LH, Ederle J, McCabe DJ, et al. Long-term risk of carotid restenosis in patients randomly assigned to endovascular treatment or endarterectomy in the Carotid and Vertebral Artery Transluminal Angioplasty Study (CAVATAS): long-term follow-up of a randomised trial. Lancet Neurol. 2009;8(10):908-917.

4. Brott TG, Hobson RW II, Howard G, et al. Stenting versus endarterectomy for treatment of carotid-artery stenosis. $N$ Engl J Med. 2010;363(1):11-23.

5. Eckstein HH, Ringleb P, Allenberg JR, et al. Results of the Stent-Protected Angioplasty versus Carotid Endarterectomy (SPACE) study to treat symptomatic stenoses at 2 years: a multinational, prospective, randomised trial. Lancet Neurol. 2008;7(10):893-902.

6. Gurm HS, Yadav JS, Fayad P, et al. Long-term results of carotid stenting versus endarterectomy in high-risk patients. $N$ Engl J Med. 2008;358(15):1572-1579.

7. Lal BK, Beach KW, Roubin GS, et al. Restenosis after carotid artery stenting and endarterectomy: a secondary analysis of CREST, a randomised controlled trial. Lancet Neurol. 2012;11(9):755-763.

8. Hellings WE, Moll FL, De Vries JP, et al. Atherosclerotic plaque composition and occurrence of restenosis after carotid endarterectomy. JAMA. 2008;299(5):547-554.

9. Katano H, Yamada K. Analysis of calcium in carotid plaques with Agatston scores for appropriate selection of surgical intervention. Stroke. 2007;38(11):3040-3044.

10. Katano H, Mase M, Nishikawa Y, et al. Analysis of recurrent stenosis after carotid endarterectomy featuring primary plaque calcification. Neurosurgery. 2017;80(6):863-870.

11. Grant EG, Benson CB, Moneta GL, et al. Carotid artery stenosis: gray-scale and Doppler US diagnosis-Society of Radiologists in Ultrasound Consensus Conference. Radiology. 2003;229(2):340-346.

12. Geroulakos G, Ramaswami G, Nicolaides A, et al Charac- terization of symptomatic and asymptomatic carotid plaques using high-resolution real-time ultrasonography. Br J Surg. 1993;80(10):1274-1277.

13. Salem MK, Bown MJ, Sayers RD, et al. Identification of patients with a histologically unstable carotid plaque using ultrasonic plaque image analysis. Eur J Vasc Endovasc Surg. 2014;48(2):118-125.

14. Biasi GM, Froio A, Diethrich EB, et al. Carotid plaque echolucency increases the risk of stroke in carotid stenting: the Imaging in Carotid Angioplasty and Risk of Stroke (ICAROS) study. Circulation. 2004;110(6):756-762.

15. Wang X, Yang B, Ma Y, et al. Comparison of monitoring of cerebral blood flow by c-FLOW and transcranial doppler in carotid endarterectomy. World Neurosurg. 2018;111:e686-e692.

16. Schulte-Altedorneburg G, Droste DW, Haas N, et al. Preoperative B-mode ultrasound plaque appearance compared with carotid endarterectomy specimen histology. Acta Neurol Scand. 2000;101(3):188-194.

17. Spanos K, Tzorbatzoglou I, Lazari P, et al. Carotid artery plaque echomorphology and its association with histopathologic characteristics. J Vasc Surg. 2018;68(6):1772-1780.

18. Hellings WE, Moll FL, de Vries JP, et al. Histological characterization of restenotic carotid plaques in relation to recurrence interval and clinical presentation: a cohort study. Stroke. 2008;39(3):1029-1032.

19. Takami H, Mizutani T, Ota T, Yuyama R. Spontaneous regression of restenosis after CEA: significance of preoperative plaque characteristics under duplex ultrasound; clinical investigation. Acta Neurochir (Wien). 2014;156(1):63-67.

20. Crawford RS, Chung TK, Hodgman T, et al. Restenosis after eversion vs patch closure carotid endarterectomy. $J$ Vasc Surg. 2007;46(1):41-48.

21. Vrijenhoek JE, de Borst GJ, den Ruijter HM, et al. A lipid-poor plaque and asymptomatic status in women are associated with higher peak systolic velocity on duplex ultrasound after carotid endarterectomy. Atherosclerosis. 2014;237(2):677-683.

22. Hudorovic N, Lovricevic I, Hajnic H, Ahel Z. Postoperative internal carotid artery restenosis after local anesthesia: presence of risk factors versus intraoperative shunt. Interact Cardiovasc Thorac Surg. 2010;11(2):182-184.

\section{Disclosures}

The authors report no conflict of interest concerning the materials or methods used in this study or the findings specified in this paper.

\section{Author Contributions}

Conception and design: Hua, Li. Acquisition of data: Li, Zhao. Analysis and interpretation of data: Li, B Liu. Drafting the article: Li. Critically revising the article: Hua, B Liu, Y Liu, Gao, Jia. Reviewed submitted version of manuscript: Y Liu. Approved the final version of the manuscript on behalf of all authors: Hua. Statistical analysis: Li. Administrative/technical/material support: Hua, B Liu, Jiao.

\section{Supplemental Information \\ Online-Only Content}

Supplemental material is available with the online version of the article.

Supplemental Table 1. https://thejns.org/doi/suppl/10.3171/ 2020.2.JNS193397.

\section{Correspondence}

Yang Hua: Xuanwu Hospital, Capital Medical University, Beijing, China.dryanghua99@163.com. 